\title{
Peptide Szeto-Schiller 31 ameliorates doxorubicin-induced cardiotoxicity by inhibiting the activation of the p38 MAPK signaling pathway
}

\author{
LI ZHANG ${ }^{1,2^{*}}$, MENGWEN FENG $^{2 *}$, XUEJUN WANG ${ }^{2 *}$, HAO ZHANG $^{3}$, \\ JINGJING DING ${ }^{4}$, ZIJIE CHENG ${ }^{2}$ and LINGMEI QIAN ${ }^{1,2}$

\begin{abstract}
${ }^{1}$ Department of Cardiology, Tongren Hospital, Shanghai Jiao Tong University School of Medicine, Shanghai 200336;
${ }^{2}$ Department of Cardiology, The First Affiliated Hospital of Nanjing Medical University, Nanjing, Jiangsu 210029;

${ }^{3}$ Department of Internal Medicine, The Affiliated Drum Tower Hospital of Nanjing University Medical School, Nanjing, Jiangsu 210008; ${ }^{4}$ Department of General Practice, Tongren Hospital, Shanghai Jiao Tong University
\end{abstract} \\ School of Medicine, Shanghai 200336, P.R. China
}

Received October 23, 2020; Accepted February 8, 2021

DOI: $10.3892 / \mathrm{ijmm} .2021 .4896$

\begin{abstract}
Oxidative stress serves a key role in doxorubicin (DOX)-induced cardiotoxicity. The peptide Szeto-Schiller (SS)31 is an efficacious antioxidant with the capacity to reduce mitochondrial reactive oxygen species (ROS) levels and scavenge free radicals. Although SS31 is involved in the pathophysiological process of various cardiovascular diseases, the role of SS31 in DOX-induced cardiotoxicity remains unclear. To explore the effects of SS31 in DOX-induced cardiotoxicity, the present study first constructed DOX-induced cardiotoxicity models, in which $\mathrm{H} 9 \mathrm{c} 2$ cells were incubated with $1 \mu \mathrm{M}$ DOX for $24 \mathrm{~h}$ and C57BL/6 mice were administered DOX $(20 \mathrm{mg} / \mathrm{kg}$ cumulative dose). The results of various assays in these models demonstrated that SS31 exhibited a cardioprotective effect in vitro and in vivo by attenuating the level of ROS, stabilizing the mitochondrial membrane potential and ameliorating myocardial apoptosis as well as fibrosis following treatment with DOX. Mechanistically, the results of the present study revealed that the p38 MAPK signaling pathway was inhibited by SS31 in DOX-treated H9c2 cells, which was associated with the cardioprotective function
\end{abstract}

Correspondence to: Professor Lingmei Qian, Department of Cardiology, Tongren Hospital, Shanghai Jiao Tong University School of Medicine, 1111 Xianxia Road, Shanghai 200336, P.R. China

E-mail: 1mqian@njmu.edu.cn

Dr Zijie Cheng, Department of Cardiology, The First Affiliated Hospital of Nanjing Medical University, 300 Guangzhou Road, Nanjing, Jiangsu 210029, P.R. China

E-mail: zjcheng@njmu.edu.cn

${ }^{*}$ Contributed equally

Key words: doxorubicin, cardiotoxicity, Szeto-Schiller 31 peptide, reactive oxygen species, p38 MAPK of SS31. In addition, P79350, a selective agonist of p38 MAPK, reversed the protective effects of SS31. Taken together, these results demonstrated the effects of SS31 on ameliorating DOX-induced cardiotoxicity and indicated its potential as a drug for the treatment of DOX-induced cardiotoxicity.

\section{Introduction}

Doxorubicin is an anthracycline anticancer drug that is clinically used to treat several types of hematological cancer and solid tumors, including leukemia, breast, endometrial and bladder cancer (1). However, the strong anticancer effect of DOX is often accompanied by a range of side effects, such as bone marrow suppression, stomatitis, fatigue and alopecia; the most severe side effect is dose-dependent cardiotoxicity, which limits the clinical use of $\operatorname{DOX}(2,3)$. A previous study has reported that patients treated with a cumulative dose of $400 \mathrm{mg} / \mathrm{m}^{2}$ DOX present an increased risk of cardiotoxicity; this risk is increased by 26 and $48 \%$ at 550 and $700 \mathrm{mg} / \mathrm{m}^{2}$ compared with that before starting the DOX treatment, respectively (4). Myocardial oxidative stress, mitochondrial impairment, intracellular calcium dysregulation and extracellular matrix remodeling are the main molecular mechanisms underlying DOX-induced cardiotoxicity (5). Reactive oxygen species (ROS) produced by oxidative stress is a major mechanism among those that have been elucidated to date, accumulating in the myocardium, causing apoptosis and further leading to cardiac dysfunction and eventually heart failure (6). The development of drugs to treat and prevent the cardiotoxicity induced by DOX has recently attracted attention (7). Dexrazoxane, the most effective agent for alleviating the DOX-induced cardiotoxicity, is converted into a chelating agent in cells and interferes with the formation of free radicals mediated by iron; however, dexrazoxane may diminish the anticancer effects and potentiate certain side effects of DOX (8). Therefore, it is necessary to find a more effective strategy to treat DOX-induced cardiotoxicity, for which oxidative stress may be a potential target. 
The peptide Szeto-Schiller (SS)-31 (SS31; H-D-Arg-DmtLys-Phe-NH2) is an efficacious antioxidant that has the capacity to reduce mitochondrial ROS and scavenge free radicals (9). It has been reported that SS31 is involved in the pathophysiological processes of a variety of cardiovascular diseases, including protection of the myocardium from ischemia-reperfusion (I/R) injury by reducing inflammation, oxidative stress, apoptosis and fibrosis (10), reduction of proteomic alterations in heart failure by preserving mitochondrial function (11), prevention of sepsis-induced cardiac damage by suppressing the inflammatory response and maintaining mitochondrial membrane potential (12), and amelioration of angiotensin II-induced cardiomyopathy by decreasing the level of ROS (13). However, whether SS31 serves a protective role in DOX-induced cardiotoxicity remains to be elucidated.

The mitogen-activated protein kinase (MAPK) pathways serve a crucial role in the regulation of apoptosis. Extracellular-regulated kinase (ERK) 1/2, p38 MAPK and c-Jun N-terminal kinase (JNK) are three major members of MAPKs and exert antiapoptotic or proapoptotic effects in different cell types and contexts (14). For example, MAPK serves a protective role in I/R-induced cardiac myocyte apoptosis and in isolated perfused hearts that have undergone reperfusion injury, whereas p38 and JNK promote apoptosis in cardiomyocytes subjected to I/R (15). Fasudil suppresses isoproterenol-induced heart failure by inhibiting the activation of JNK and the nuclear translocation of MAPK (16). Therefore, the present study focused on the development of strategies to improve DOX-induced cardiotoxicity with MAPK as a target.

The present aimed to determine the therapeutic effects of SS31 on DOX-induced cardiotoxicity, and we hypothesized that the administration of SS31 may provide a new insight into therapeutic strategies for the treatment of DOX-induced cardiotoxicity.

\section{Materials and methods}

Peptide synthesis and administration. The peptide SS31 (H-D-Arg-Dmt-Lys-Phe-NH2) was synthesized by Shanghai Science Peptide Biological Technology Co., Ltd. The peptide crystal was dissolved in sterile double-distilled water and diluted to 10,20 and $50 \mu \mathrm{mol} / 1$.

Cell culture and treatment. $\mathrm{H} 9 \mathrm{c} 2$, a rat cardiomyocyte-derived cell line, was acquired from The Cell Bank of Type Culture Collection of The Chinese Academy of Sciences. H9c2 cells were cultured in Dulbecco's modified Eagle's medium (DMEM; Gibco; Thermo Fisher Scientific, Inc.) supplemented with $10 \%$ fetal bovine serum (Gibco; Thermo Fisher Scientific, Inc.) and $1 \%$ penicillin and streptomycin (Wisent, Inc.) in a $95 \%$ air and $5 \% \mathrm{CO}_{2}$ atmosphere at $37^{\circ} \mathrm{C}$.

The H9c2 cell toxicity model was induced by DOX (cat. no. CAS 25316-40-9; Target Molecule Corp.). The H9c2 cells at $80 \%$ confluence were preincubated with the SS31 peptide or vehicle for $2 \mathrm{~h}$ at $37^{\circ} \mathrm{C}$, followed by the addition of $1 \mu \mathrm{M}$ DOX to the medium for $24 \mathrm{~h}$ at $37^{\circ} \mathrm{C}$ (17). Cells were designated into six groups: i) Control group; ii) SS31 (50 $\mu \mathrm{mol} / 1)$ treatment group; iii) DOX treatment group; iv) DOX and SS31 (10 $\mu \mathrm{mol} / \mathrm{l})$ cotreatment group; v) DOX and SS31 (20 $\mu \mathrm{mol} / \mathrm{l})$ cotreatment group; vi) DOX and SS31 (50 $\mu \mathrm{mol} / \mathrm{l})$ cotreatment group. The
SS31 peptide was added to the culture supernatant $2 \mathrm{~h}$ prior to DOX treatment. A selective agonist of p38 MAPK P79350 (50 $\mu \mathrm{M}$; Invitrogen; Thermo Fisher Scientific, Inc.) was used to activate the $\mathrm{p} 38$ signaling pathway. P79350 was added to the cell culture medium for $24 \mathrm{~h}$ at $37^{\circ} \mathrm{C}$.

Crystal violet staining assay. Following treatment, H9c2 cells were seeded in 6 -well plates at $2 \times 10^{5}$ cells/well, washed with PBS twice to remove dead cells and fixed with $4 \%$ paraformaldehyde at room temperature (RT) for $30 \mathrm{~min}$. Subsequently, the cells were washed again with PBS and stained with $0.1 \%$ crystal violet (Beyotime Institute of Biotechnology) solution for $30 \mathrm{~min}$ at RT in the dark. The dye was aspirated, and the cells were washed twice with PBS and air-dried naturally at RT for image capture under light microscopy (x200). Subsequently, the dye was solubilized with $33 \%$ acetic acid solution for $10 \mathrm{~min}$ at RT, and the absorbance was quantified at $570 \mathrm{~nm}$ using a microplate reader.

Cell survival analysis. H9c2 cells were seeded in 6-well plates at $2 \times 10^{5}$ cells/well. Following DOX and peptide treatment, the cells were digested with trypsin-EDTA solution for $3 \mathrm{~min}$ at $37^{\circ} \mathrm{C}$ and pipetting. Trypan blue staining was used to assess the cell survival rates. Briefly, the cells were resuspended with $1 \mathrm{ml}$ PBS, and $100 \mu \mathrm{l}$ of the cell suspension was added to $100 \mu \mathrm{l}$ trypan blue solution and stained for $3 \mathrm{~min}$. The number of cells was counted in the four squares of the hemocytometer under a Zeiss light microscope (magnification, x100). The following formula was used for the cell survival rate: Cell survival rate $(\%)=($ no. of living cells/no. of total cells) $x 100 \%$.

Analysis of cell viability. Cell viability was assessed using the CCK-8 Assay kit (Beyotime Institute of Biotechnology) according to the manufacturer's instructions. H9c2 cells were seeded in 96-well plates at $1 \times 10^{4}$ cells/well and cultured to adherence, followed by SS31 and DOX treatment as aforementioned. A total of $10 \mu \mathrm{l} \mathrm{CCK}-8$ reagent was supplemented into each well, and the cells were incubated in the dark for $2 \mathrm{~h}$ at $37^{\circ} \mathrm{C}$. The absorbance was measured using a microplate reader at $450 \mathrm{~nm}$, and the cell viability was calculated based on the absorbance.

Lactate dehydrogenase ( $L D H)$ determination. The levels of LDH were detected by the LDH Release Assay kit (Beyotime Institute of Biotechnology). The reaction solution was prepared according to the manufacturer's instructions. The H9c2 cell supernatant $(120 \mu \mathrm{l} /$ well $)$ was collected by centrifugation at $400 \mathrm{x} \mathrm{g}$ for $5 \mathrm{~min}$ at RT and mixed with the reaction solution (60 $\mu \mathrm{l} /$ well), and the mixtures were added into 96 -well plates. The plates were wrapped in tin foil and incubated for $30 \mathrm{~min}$ at RT on the shaker. Finally, the absorbance was detected with a microplate reader at $490 \mathrm{~nm}$ wavelength.

Analysis of ROS production. The level of intracellular total ROS was assessed by 2',7'-dichlorofluorescin diacetate (DCFH-DA) using a Reactive Oxygen Species Assay kit (Beyotime Institute of Biotechnology). Briefly, H9c2 cells were seeded in 6-well plates at $2 \times 10^{5}$ cells/well and treated as aforementioned when the cells had grown to $80 \%$ confluence. DCFH-DA was diluted to $10 \mu \mathrm{M}$ in serum-free DMEM and added into the medium 
( $1 \mathrm{ml} /$ well), and the cells were incubated at $37^{\circ} \mathrm{C}$ away from light for $20 \mathrm{~min}$. Subsequently, the cells were washed with PBS thrice to remove the residual DCFH-DA and examined in at $\geq 3$ fields per sample under a laser scanning confocal microscope (magnification, $x 100)$. The density of ROS fluorescence was examined by ImageJ software 1.26 (National Institutes of Health).

JC-1 mitochondrial membrane potential determination. The JC-1 Mitochondrial Membrane Potential Assay kit (Beyotime Institute of Biotechnology) was used to analyze mitochondrial injury according to the manufacturer's instructions. Briefly, $\mathrm{H} 9 \mathrm{c} 2$ cells were seeded in 6 -well plates at $2 \times 10^{5}$ cells/well, washed with PBS and incubated with JC-1 solution for $10 \mathrm{~min}$ at $37^{\circ} \mathrm{C}$. The cells were washed with the dilution buffer and analyzed in $\geq 3$ fields per sample under a laser scanning confocal microscope (magnification, x100).The density of JC-1 fluorescence was examined by ImageJ software.

Western blotting. H9c2 cells were treated as aforementioned and lysed by RIPA protein lysis buffer (Beyotime Institute of Biotechnology) and 1\% PMSF (Beyotime Institute of Biotechnology) to extract the total protein. The protein concentrations were determined using the Bicinchoninic Acid Protein Assay kit (Beyotime Institute of Biotechnology). The proteins $(20 \mu \mathrm{g} /$ lane $)$ were separated by $10 \%$ SDS-PAGE and transferred to PVDF membranes (MilliporeSigma). The membranes were blocked with skimmed milk (5\%) for $2 \mathrm{~h}$ at RT and incubated with the primary antibodies against PARP (1:1,000; cat. no. 9542), cleaved caspase-3 (1:1,000; cat. no. 9661), bax (1:1,000; cat. no. 2772), bcl-2 (1:1,000; cat. no. 4223), phosphorylated (p-)p38 (1:1,000; cat. no. 4511), p38 (1:1,000; cat. no. 8690), JNK (1:1,000; cat. no. 9255), p-JNK (1:1,000; cat. no. 9251), p-ERK (1:1,000; cat. no. 4376), ERK (1:1,000; cat. no. 4695), $\alpha / \beta$-Tubulin $(1: 2,000$; cat. no. 2148), $\beta$-actin $(1: 2,000$; cat. no. 4970) and GAPDH $(1: 2,000$; cat. no. 2118) (all Cell Signaling Technology, Inc.) at $4^{\circ} \mathrm{C}$ overnight. Notably, the internal control antibody against $\alpha / \beta$-tubulin produced a nonspecific faint band $(18,19)$. Subsequently, PVDF membranes were washed three times for 10 min each time with TBS with $0.1 \%$ Tween-20 buffer and incubated with horseradish peroxidase-conjugated anti-rabbit secondary antibodies (1:3,000; cat. no. 7074; Cell Signaling Technology, Inc.) for $1 \mathrm{~h}$ at RT. The immunoreactive protein bands were detected using an enhanced chemiluminescent substrate (cat. no. SQ101; Epizyme, Inc.). The protein expression levels were quantified according to their grey values determined using ImageJ software.

In vivo experiment. All animal experiments were carried out in accordance with the Guide for the Care and Use of Laboratory (20) and approved by the Institutional Animal Care and Use Committee of Nanjing Medical University (approval no. IACUC-1903030; Nanjing, China). A total of 48 male C57BL/6 mice (6 weeks old; weight, 16-20 g) were obtained from Shanghai SLAC laboratory animal corporation and maintained under a $12: 12-\mathrm{h}$ light/dark cycle at $22-26^{\circ} \mathrm{C}$ with a relative humidity of $40-50 \%$ and ad libitum food and water. Following one week of adjustable feeding, the animals were randomly assigned to the following groups: i) Vehicle; ii) SS31; iii) DOX; and iv) DOX + SS31 treated animals. DOX was administrated by intraperitoneal injection at $5 \mathrm{mg} / \mathrm{kg}$ weekly, and the final cumulative dose was $20 \mathrm{mg} / \mathrm{kg}$ (21). SS31 (2.5 mg/ $\mathrm{kg})$ was injected into the tail vein weekly, and the final cumulative dose was $10 \mathrm{mg} / \mathrm{kg}$ (22). The mice that were not treated with SS31 or DOX received the equal volumes of saline. Following the treatment, the mice were maintained alive for one week. The mice were anesthetized with $1.5 \%$ isoflurane inhalation; the depth of anesthesia was evaluated by the immobility and the absence of righting reflex, and echocardiography was used to detect mouse cardiac function. Following euthanasia by carbon dioxide asphyxia (30\% chamber volume/min), the hearts were removed rapidly and harvested to prepare paraffin sections for Masson and wheat germ agglutinin (WGA) staining or to isolate the mitochondria. The blood $(1 \mathrm{ml})$ was collected from the abdominal aorta, and the serum was obtained by centrifugation $\left(1,200 \times \mathrm{g}, 20 \mathrm{~min}, 4^{\circ} \mathrm{C}\right)$.

Echocardiography analysis. For the evaluation of cardiac function, the mice were anesthetized with $1.5 \%$ isoflurane, and echocardiography was performed using a Vevo 2100 High Resolution Imaging System (VisualSonics, Inc.). Cardiac contractile function was examined by echocardiography in conscious, gently restrained mice using a Vevo 2100 system (MS400C probe). The main measured indicators included ejection fraction (EF) and fractional shortening (FS). Other echocardiographic parameters included left ventricular end-systolic diameter (LVEDs), left ventricular end-diastolic diameter (LVEDd), left ventricular end-systolic volume (LVESV) and left ventricular end-diastolic volume (LVEDV). FS was calculated as follows: FS $(\%)=[(L V E D d-L V E D s) / L V E D d] \times 100 ;$ EF was calculated as follows: $\mathrm{EF}(\%)=[(\mathrm{LVEDV}-\mathrm{LVESV}) / \mathrm{LVEDV}] \times 100$, where LVEDV=7 $\times \mathrm{LVEDd}^{3} /(2.4+\mathrm{LVEDd})$ and LVESV= $7 \times \mathrm{LVEDs}^{3} /(2.4+$ LVEDs $)$.

WGA and Masson staining. The hearts were harvested and fixed in $4 \%$ buffered formaldehyde for $48 \mathrm{~h}$ at RT. After embedding in paraffin and sectioning, $5-\mu \mathrm{m}$ sections were stained with fluorescein isothiocyanate-conjugated WGA (cat. no. L4895; Sigma-Aldrich; Merck KGaA) staining according to the manufacturer's instructions. Digital images ( $\geq 3$ fields) were captured using a laser scanning confocal microscope (magnification, $x 400)$. A quantitative digital image analysis system Image-Pro Plus 6.0 (Media Cybernetics, Inc.) was used to measure the cross-sectional area of cardiomyocytes. To assess cardiac fibrosis, the 5- $\mu \mathrm{m}$ sections were stained with Masson's trichrome (cat. no. G1340; Beijing Solarbio Science \& Technology Co., Ltd.) according to the manufacturer's instructions. Each stained section was observed under a microscope (magnification, $\mathrm{x} 400$ ), and ImageJ software was used to evaluate histopathological damage.

LDH, superoxide dismutase (SOD), malondialdehyde (MDA) and glutathione peroxidase (GSH-PX) measurement. The serum LDH concentrations were measured using an ELISA kit (cat. no. J2380; Promega Corporation) according the manufacturer's instructions. A total of $40 \mathrm{mg}$ heart tissues were harvested to isolate the mitochondria. Briefly, tissues were incised completely in $500 \mu \mathrm{l}$ PBS and centrifuged at 
A

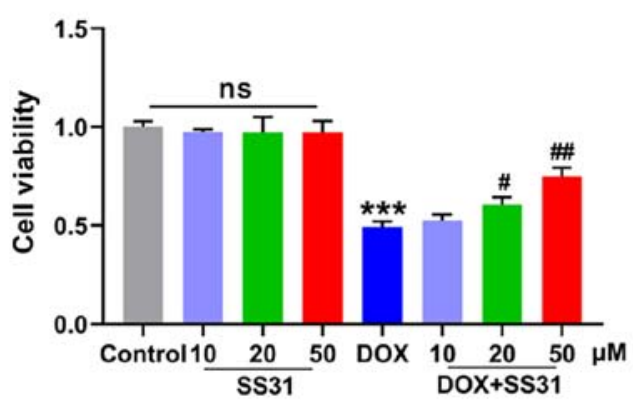

B

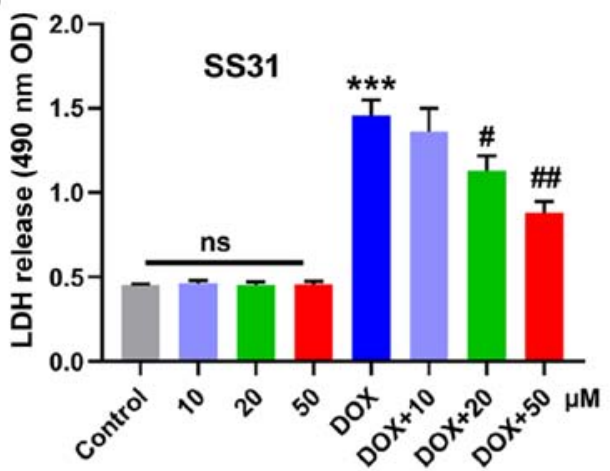

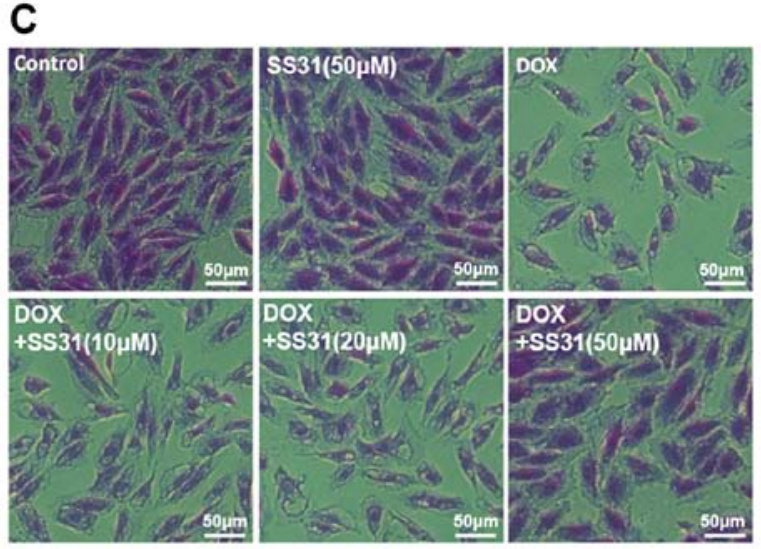

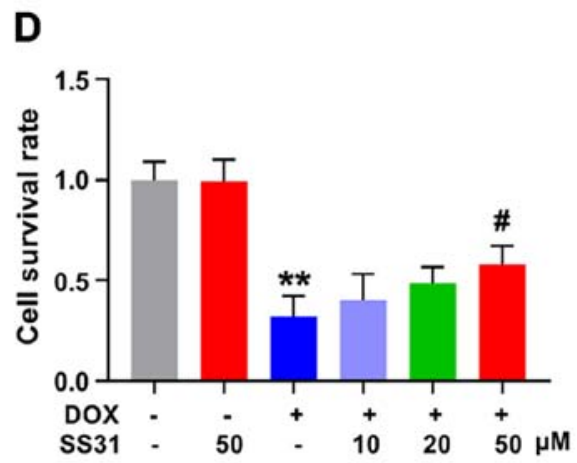

Figure 1. SS31 treatment attenuates DOX-induced inhibition of H9c2 cell survival. (A) The cell viability was determined in H9c2 cells. (B) LDH release level was measured in $\mathrm{H} 9 \mathrm{c} 2$ cells supernatant. (C and D) Crystal violet staining and quantitative analysis of living cells. Magnification, $\mathrm{x} 200$. ${ }^{* *} \mathrm{P}<0.01$ and ${ }^{* * *} \mathrm{P}<0.001$ vs. control; ${ }^{\#} \mathrm{P}<0.05$ and ${ }^{\# \#} \mathrm{P}<0.01$ vs. DOX; ns, not statistically significant. DOX, doxorubicin; SS31, Szeto-Schiller 31 peptide; LDH, lactate dehydrogenase; OD, optical density.

$800 \mathrm{x} \mathrm{g}$ for $5 \mathrm{~min}$. After centrifuge, $500 \mu \mathrm{l}$ isolation reagent were added to purify the mitochondria. The SOD, MDA and GSH-PX were determined using commercially available kits (cat. nos. A001-3, A003-1 and A005-1, respectively; Nanjing Jiancheng Bio Company) according to the manufacturer's instructions by measuring absorbance at 450, 532 and $412 \mathrm{~nm}$, respectively, with a microplate reader.

Statistical analysis. Data are presented as the mean \pm standard deviation. All experimental data were analyzed by GraphPad Prism 8 software (GraphPad Software, Inc.). The differences amongst multiple groups were analyzed by one-way ANOVA with a post hoc Bonferroni's multiple comparisons test. Mouse survival was analyzed by the log-rank test. $\mathrm{P}<0.05$ was considered to indicate a statistically significant difference.

\section{Results}

SS31 treatment attenuates DOX-induced inhibition of $\mathrm{H} 9 \mathrm{c} 2$ cell survival. To investigate the function of SS31, the present study first treated H9c2 cells with SS31 to identify the location of SS31. As presented in Fig. S1A, SS31 entered the cells. The changes in the $\mathrm{H} 9 \mathrm{c} 2$ cell survival following treatment with various concentrations of $\operatorname{DOX}(0,0.1,0.25,0.5,1.0$, $2.5,5.0$ and $10.0 \mu \mathrm{M})$ for various durations $(0,6,12,24$ and $36 \mathrm{~h}$ ) were first assessed prior to demonstrating the function of SS31 in DOX-induced cardiotoxicity. As demonstrated in Fig. S1B and C, a stable in vitro cardiotoxicity model was established by $1-\mu \mathrm{M}$ DOX treatment for $24 \mathrm{~h}$. The results of subsequent experiments demonstrated that SS31 alleviated DOX-induced H9c2 cell damage and had no effect on H9c2 cells without DOX treatment, as revealed by the cell viability and $\mathrm{LDH}$ release assays (Fig. 1A and B). In addition, crystal violet staining confirmed that SS31 (20 and $50 \mu \mathrm{M})$ improved the survival rate of DOX-treated H9c2 cells (Fig. 1C and D). These results indicated that SS31 may exert protection from DOX-induced H9c2 cell damage.

SS31 attenuates DOX-induced apoptosis in H9c2 cells. To further explore the function of SS31 in DOX-induced myocardial injury, the expression levels of apoptosis-associated proteins were evaluated in H9c2 cells. Decreased PARP activation, cleaved caspase- 3 and bax levels and increased bcl-2 protein levels were detected in cells pretreated with SS31 (20 and $50 \mu \mathrm{M})$ prior to DOX treatment compared with those in cells only exposed to DOX (Fig. 2A-E). These results suggested that SS31 attenuated DOX-induced apoptosis in H9c2 cells.

SS31 attenuates DOX-induced mitochondrial oxidative stress injury in $\mathrm{H} 9 \mathrm{c} 2$ cells. Since the accumulation of intracellular total ROS is the main biological event of DOX-induced myocardial injury (6), the total ROS accumulation in $\mathrm{H} 9 \mathrm{c} 2$ cells was examined by DCFH-DA staining. The results demonstrated that $50 \mu \mathrm{M}$ SS31 significantly decreased the level of ROS following DOX treatment in H9c2 cells compared with that in the cells treated with DOX alone (Fig. 3A and B). In addition, $50 \mu \mathrm{M}$ SS31 significantly alleviated the decrease of mitochondrial membrane potential induced by DOX, as 
A

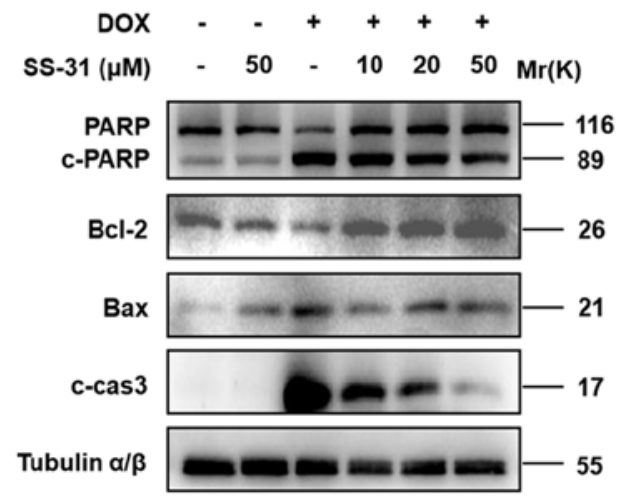

B

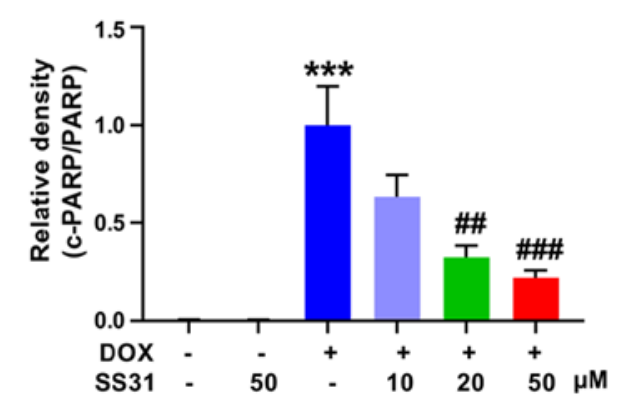

C

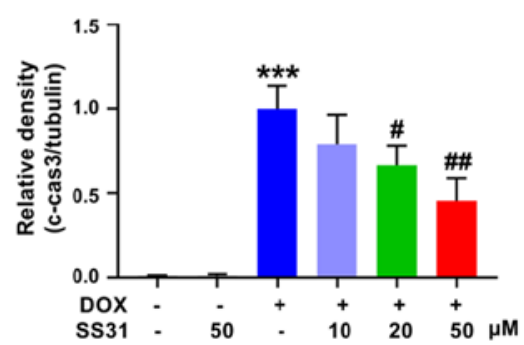

D

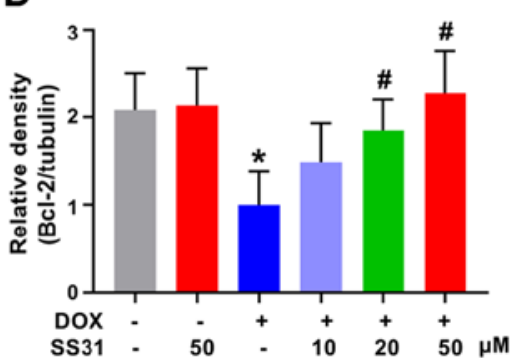

E

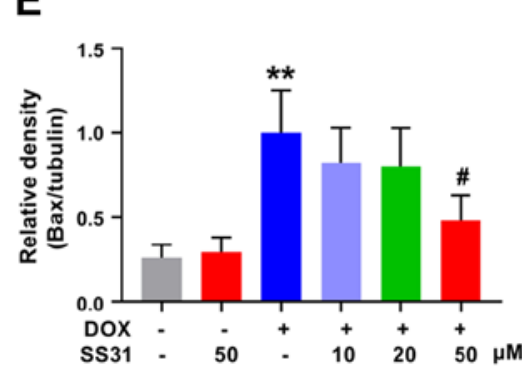

Figure 2. SS31 attenuates DOX-induced apoptosis in H9c2 cells. (A) The levels of apoptosis-related proteins (PARP, cleaved caspase-3, bax and bcl-2) were determined by western blotting. (B-E) Quantitative analysis of the relative protein levels of (B) c-PARP, (C) cleaved caspase-3, (D) bcl-2 and (E) bax. *P $<0.05$,

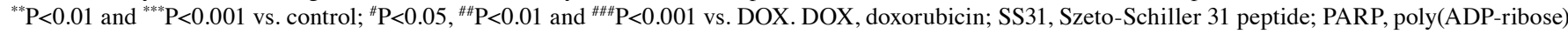
polymerase; c-, cleaved; cas3, caspase-3.

evidenced by the lower ratio of JC-1 monomer to aggregate in the SS31-pretreated cells compared with that in the cells treated with DOX alone (Fig. 3C and D).

Effects of SS31 on DOX-induced cardiac injury in vivo. To assess the effects of SS31 on DOX-induced cardiac injury in vivo, a mouse model of cardiotoxicity was constructed by an intraperitoneal injection of $20 \mathrm{mg} / \mathrm{kg}$ DOX and a tail vein injection of $10 \mathrm{mg} / \mathrm{kg} \mathrm{SS} 31$ (Fig. 4A). The results of the in vivo experiments demonstrated that SS31 protected the mouse heart from DOX-induced injury, as evidenced by improved cardiac function determined by echocardiography (Fig. 4B and C) and a lower level of cardiac fibrosis (Fig. 4D and E) in mice treated with SS31 and DOX compared with those in the DOX group. The cardiomyocyte area was evaluated by WGA staining, and the results revealed a significant decrease of the cardiomyocyte area in the DOX group compared with the control group; cotreatment with SS31 reversed this effect (Fig. 4F and G). In addition, DOX-induced cardiac injury was significantly improved in the SS31 intervention group, which exhibited a decrease in the serum LDH level compared with that in mice treated with DOX alone (Fig. 4H). Oxidative stress markers were detected to evaluate the mitochondrial oxidative stress injury; the results demonstrated that SS31 attenuated the DOX-induced mitochondrial oxidative stress injury, as indicated by the differences in the MDA, SOD and GSH-PX content between the DOX and DOX + SS31 groups (Fig. 4I-K). As presented in Fig. 4L, treatment with SS31 did not affect the survival of mice following DOX injection. These results indicated that SS31 exerted a cardioprotective effect on DOX-induced cardiac injury without improving survival in vivo.
SS31 inhibits the activation of p38 MAPK signaling in DOX-treated H9c2 cells. The present study further aimed to determine the mechanism underlying the effects of SS31 in ameliorating DOX-induced cardiotoxicity in H9c2 cells. The effects of SS31 on the expression and phosphorylation of ERK1/2, JNK and p38 were examined in DOX-treated H9c2 cells. SS31 treatment had no significant effects on the levels of p-ERK1/2 and p-JNK, and the total levels of ERK1/2 and JNK were also unchanged (Fig. 5A-D). However, the DOX-induced upregulation of p-p38 was significantly inhibited by pretreatment with SS31 in H9c2 cells (Fig. 5E and F). Thus, these results revealed that SS31 inhibited the activation of the p38 MAPK signaling pathway to attenuate the ROS accumulation and apoptosis in $\mathrm{H} 9 \mathrm{c} 2$ cells.

p38 agonist reverses the effects of SS31. To further verify that SS31 protected H9c2 cells from DOX-induced injury by regulating the p38 MAPK signaling pathway, a selective agonist of p38 MAPK, P79350, was used to activate the p38 signaling pathway. As demonstrated in Fig. 6A, compared with the SS31 + DOX group, p38 was activated when the cells were treated with P79350. A comprehensive experiment was subsequently performed. The p38 agonist reversed the inhibitory effects of SS31 on the cell damage, as evidenced by the decreased cell viability, cell survival rates and increased LDH release in DOX-induced cells cotreated with P79350 and SS31 compared with those in DOX-induced cells treated with SS31 alone (Fig. 6C-F). In addition, the mitochondrial ROS content analysis results demonstrated that P79350 completely rescued effects of SS31 on ROS (Fig. 6G and H). The levels of apoptosis-associated proteins were also assessed, and the results revealed that the levels of cleaved PARP and cleaved 
A
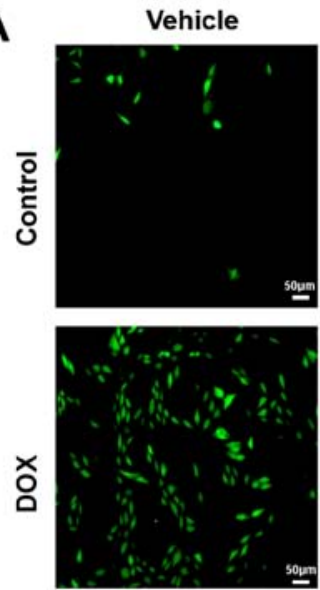

SS31 $(50 \mu \mathrm{M})$
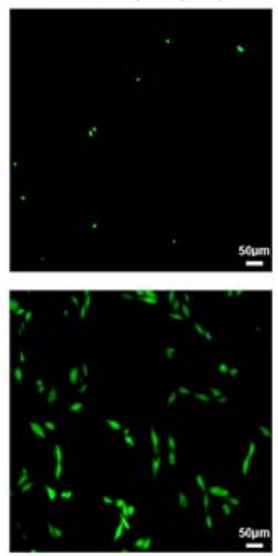

B

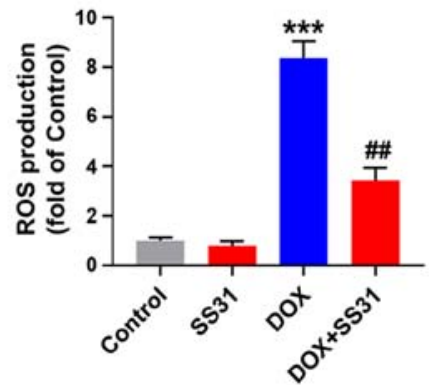

\section{C}
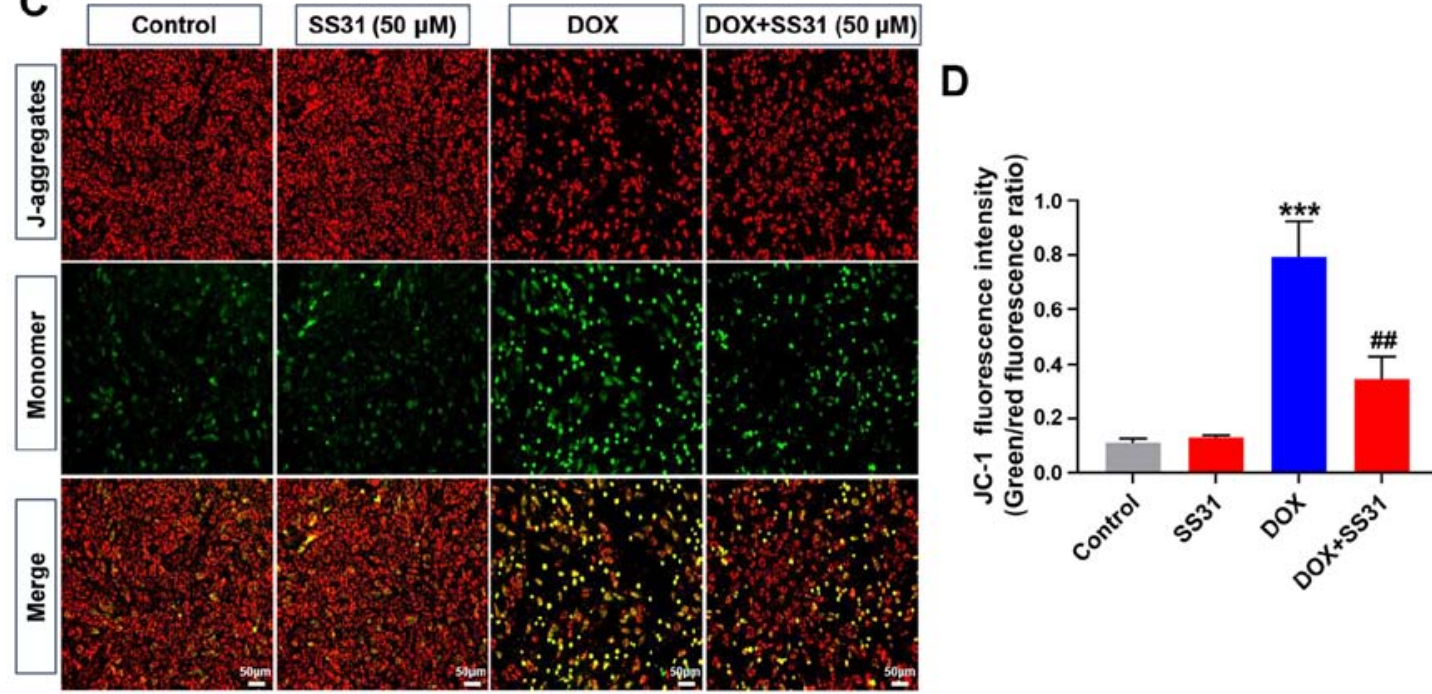

Figure 3. SS31 attenuates DOX-induced mitochondrial oxidative stress injury in H9c2 cells. (A) Representative images of DCFH-DA (green) staining in H9c2 cells used to detect intracellular ROS following treatment with $50 \mu \mathrm{M} \mathrm{SS31}$ and DOX. Magnification, x100. (B) Quantitative analysis of ROS. (C) Representative images of JC-1 fluorescence representing the mitochondrial membrane potential in H9c2 cells. Magnification, x100. (D) Quantitative analysis of JC-1 fluorescence intensity. ${ }^{* * *} \mathrm{P}<0.001$ vs. control; ${ }^{\# \#} \mathrm{P}<0.01$ vs. DOX. DOX, doxorubicin; SS31, Szeto-Schiller 31 peptide; DCFH-DA, 2',7'-dichlorofluorescin diacetate.

caspase-3 were increased in the DOX, SS31 and P79350 cotreatment group compared with those in the DOX and SS31 group, suggesting that the p38 agonist reversed the effects of SS31 (Fig. 6I-K).

\section{Discussion}

DOX is an efficient broad-spectrum antitumor drug and has been used to treat various types of cancer as a basic chemotherapy agent in clinical practice (1). However, DOX is a double-edged sword, as it also induces acute and chronic cardiac injury during or after treatment (23). Oxidative stress and apoptosis of cardiomyocytes serve important roles in DOX-induced cardiac injury $(24,25)$. In the present study, SS31 was demonstrated to exert a protective effect against DOX-induced cardiotoxicity by scavenging ROS and reducing myocardial apoptosis through the inhibition of the p38 MAPK signaling pathway. In addition, the administration of SS31 improved cardiac function and limited the extent of myocardial fibrosis in a mouse model of cardiotoxicity caused by DOX. These results suggested that SS31 may be a candidate drug for the treatment of DOX-induced cardiotoxicity.
To determine the myocardial protective effects of SS31 against DOX-induced cardiotoxicity, in vitro experiments were first performed in the present study. In previous studies, the concentration and time of the DOX in vitro model treatment were different compared with those used in the present study $(26,27)$. For example, treatment with $0.5 \mu \mathrm{M}$ DOX for $24 \mathrm{~h}$ significantly diminishes the protein expression levels of COX5A in H9c2 cells (27). MicroRNA-140-5p aggravates $5 \mu \mathrm{M}$ DOX-induced cardiotoxicity by promoting myocardial oxidative stress via targeting Nrf2 and Sirt2 in H9c2 cells (26). The present study used $\mathrm{H} 9 \mathrm{c} 2$ cells to construct a stable in vitro cardiotoxicity model by $24-\mathrm{h}$ treatment with $1 \mu \mathrm{M}$ DOX. A previous study has reported that $200 \mathrm{nM}$ DOX induces cell cycle arrest (28). However, other studies have demonstrated that $1 \mu \mathrm{M}$ DOX induces ROS accumulation and apoptosis in cardiomyocytes $(17,29)$. DOX has been reported to induce apoptosis through a number of mechanisms, including its effects on topoisomerase II, excessive production of ceramide and production of free radicals and ROS (30). In the present study, $1 \mu \mathrm{M}$ DOX induced ROS accumulation and apoptosis in cardiomyocytes. These results were consistent with the aforementioned studies and indicated that DOX induced apoptosis 
A

\begin{tabular}{|c|c|c|c|c|}
\hline DOX & $\downarrow \sqrt{5 \mathrm{mg} / \mathrm{kg}}$ & $\sqrt{5} \mathrm{mg} / \mathrm{kg}$ & $\downarrow 5 \mathrm{mg} / \mathrm{kg}$ & $\sqrt{5} \mathrm{mg} / \mathrm{kg}$ \\
\hline Week 1 & Week 2 & Week 3 & Week 4 & Week 5 \\
\hline SS31 & $\uparrow_{2.5 \mathrm{mg} / \mathrm{kg}}$ & $\uparrow 2.5 \mathrm{mg} / \mathrm{kg}$ & $\uparrow 2.5 \mathrm{mg} / \mathrm{kg}$ & $\uparrow 2.5 \mathrm{mg} / \mathrm{kg}$ \\
\hline
\end{tabular}

C
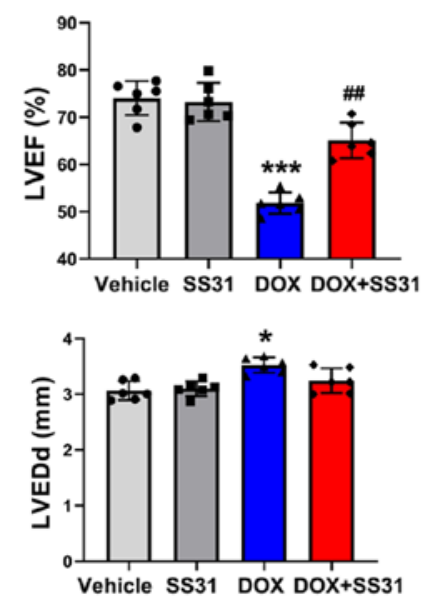

D

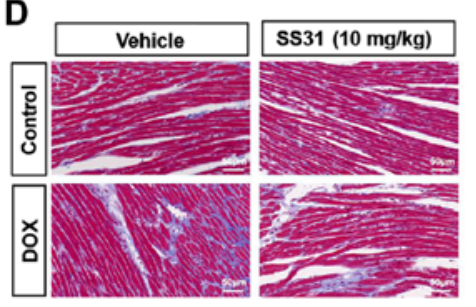

G

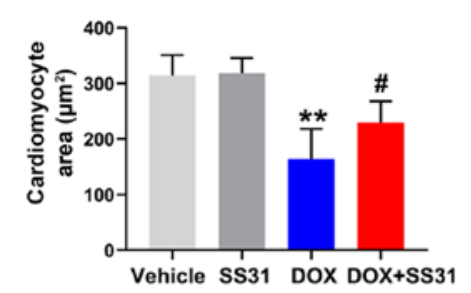

$\mathbf{J}$

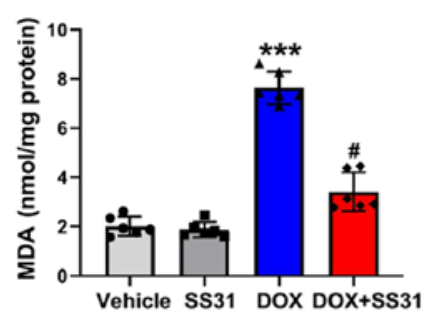

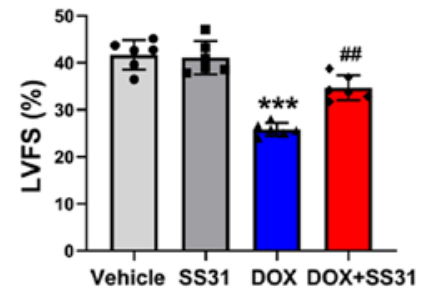

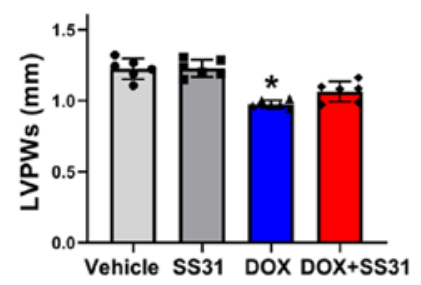

E

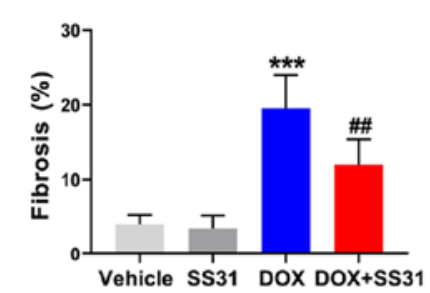

H

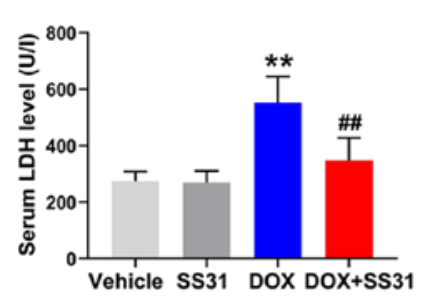

$\mathbf{K}$

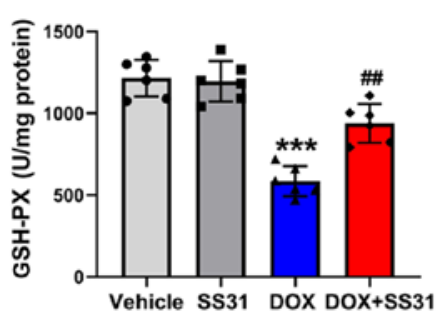

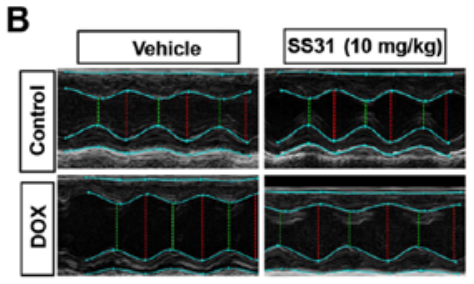
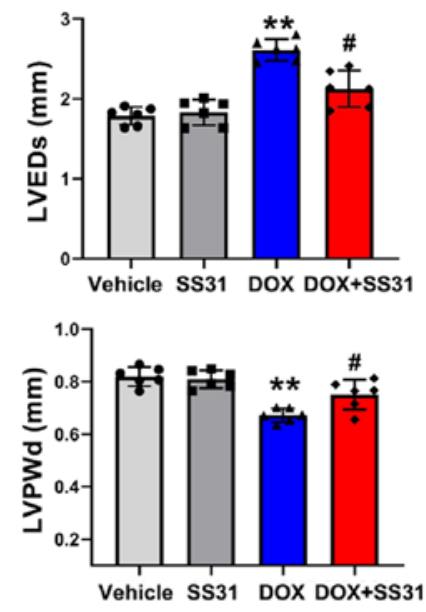

F Vehicle $\mathbf{S S 3 1 ( 1 0 \mathrm { mg } / \mathrm { kg } )}$

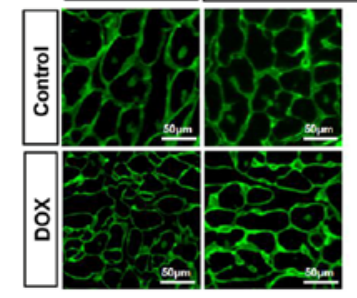

I

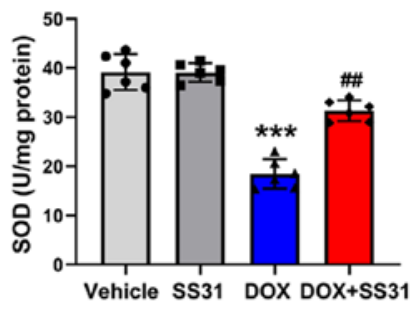

$\mathbf{L}$

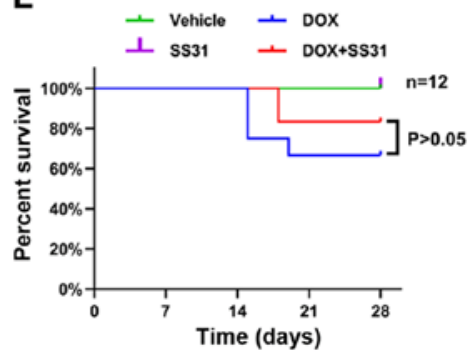

Figure 4. Effects of SS31 on DOX-induced cardiac injury in vivo. (A) Timeline of the experimental procedure for the DOX-induced mouse cardiotoxicity model. (B) Representative photographs of the echocardiography analysis. (C) Quantified data of the echocardiography analysis. (D) Masson trichrome staining. Magnification, $x 400$. (E) Quantitative analysis of fibrosis in the Masson-stained sections. (F) Representative photographs of wheat germ agglutinin staining. Magnification, $x$ 400. (G) Quantitative analysis of the cardiomyocyte area. (H) Serum LDH levels. $n=6$ mice/group. (I-K) SOD, MDA and GSH-PX levels were evaluated in mouse heart tissue samples. $n=6$ mice/group. (L) Survival of mice following DOX-induced cardiac injury. Day 0 refers to the first DOX injection. ${ }^{*} \mathrm{P}<0.05,{ }^{* *} \mathrm{P}<0.01$ and ${ }^{* * *} \mathrm{P}<0.001$ vs. vehicle; ${ }^{*} \mathrm{P}<0.05$ and ${ }^{\# \#} \mathrm{P}<0.01$ vs. DOX. DOX, doxorubicin; SS31, Szeto-Schiller 31 peptide; LDH, lactate dehydrogenase; SOD, superoxide dismutase; MDA, malondialdehyde; GSH-PX, glutathione peroxidase; EF, ejection fraction; FS, fractional shortening; LVEDs, left ventricular end-systolic diameter; LVEDd, left ventricular end-diastolic diameter; LVESV, left ventricular end-systolic volume; LVEDV, left ventricular end-diastolic volume.

in $\mathrm{H} 9 \mathrm{c} 2$ cells. Subsequently, the results of the present study demonstrated that pretreatment with SS31 improved the inhibition of cell viability induced by DOX. This effect was concentration-dependent; in addition $50 \mu \mathrm{M} \mathrm{SS31}$ did not 
A

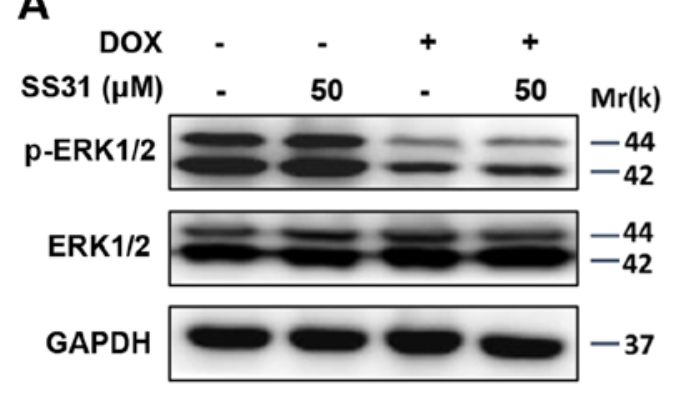

C
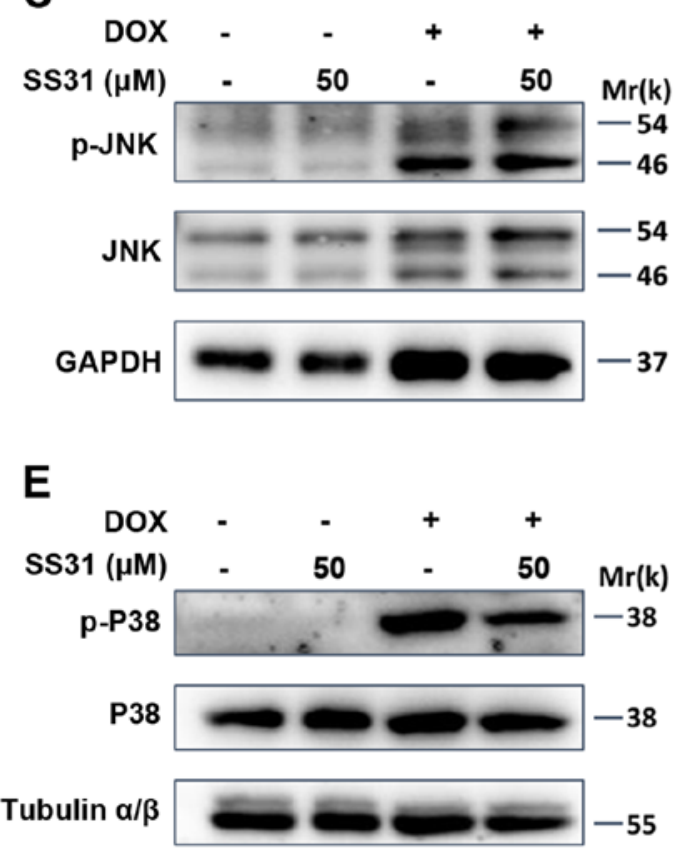

B

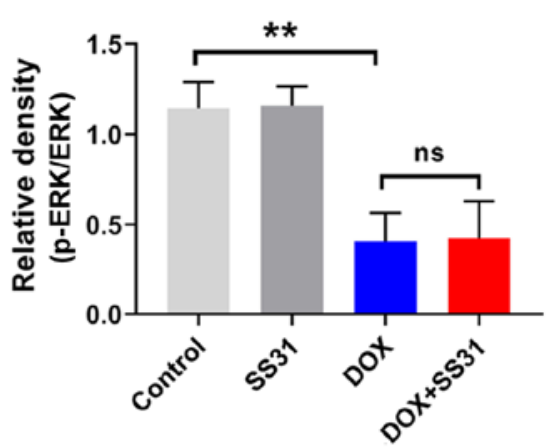

D

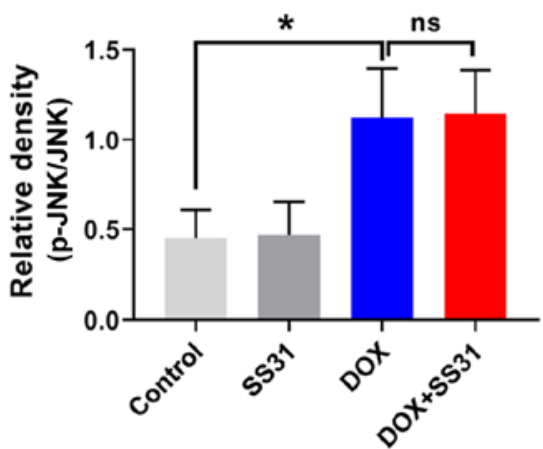

$\mathbf{F}$

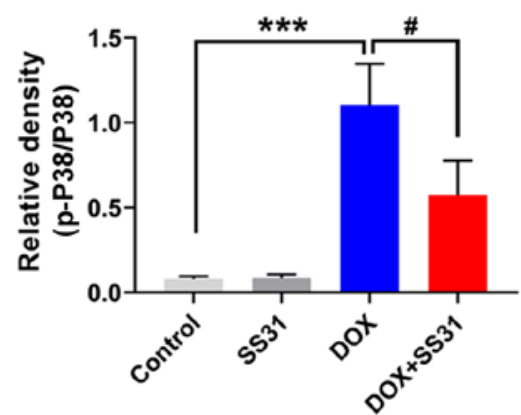

Figure 5. SS31 treatment inhibits the activation of p38 MAPK signaling in DOX-treated H9c2 cells. (A) The protein levels of p-ERK1/2 and ERK1/2 were determined by western blotting. (B) Quantitative analysis of the relative protein levels of p-ERK1/2. (C) The protein levels of p-JNK and JNK were determined by western blotting. (D) Quantitative analysis of the relative protein levels of p-JNK. (E) The protein levels of p-p38 and p38 were determined by western blotting. (F) Quantitative analysis of the relative protein levels of p-p38. ${ }^{*} \mathrm{P}<0.05,{ }^{* * *} \mathrm{P}<0.01$ and ${ }^{* * * *} \mathrm{P}<0.001$ vs. control; ${ }^{*} \mathrm{P}<0.05$ vs. DOX; ns, not statistically significant. p-, phosphorylated; DOX, doxorubicin; SS31, Szeto-Schiller 31 peptide.

exert any toxic effects on $\mathrm{H} 9 \mathrm{c} 2$ cells. Consistent with these results, crystal violet staining confirmed that SS31 improve the survival rate of DOX-treated H9c2 cells. These results suggested that SS31 may exert myocardial protection.

Although a number of mechanisms are involved in DOX-induced cardiotoxicity, oxidative stress, defined as an imbalance between the production of ROS and antioxidant reactions, is recognized as the primary mechanism (6). DOX preferentially localizes to the anionic phospholipid cardiolipin on the mitochondrial inner membrane, where it causes organelle toxicity by producing superabundant ROS (31). Supraphysiologic ROS not only contributes to mitochondrially injury via inactivation of iron-sulfur proteins and stimulation of lipid peroxidation, but also impairs macromolecules in the cells, such as nucleic acids, proteins and phospholipids, thus leading to the structural collapse of organelles and apoptosis $(32,33)$. Previous studies have reported that SS31 protects cells from oxidative stress by restoring mitochondrial function (34). SS31 exhibits potent protective effects in numerous diseases, such as diabetic nephropathy (35), Alzheimer's disease (36), subarachnoid hemorrhage (37) and Huntington's disease (38). SS31 has also been demonstrated to exhibit high efficacy in several types of cardiovascular diseases, including myocardial I/R injury, heart failure and hypertensive cardiomyopathy (9). In the present study, compared with the DOX group, SS31 reduced the release of LDH. In addition, $50 \mu \mathrm{M} \mathrm{SS} 31$ markedly decreased the levels of ROS and maintained the mitochondrial membrane potential following DOX treatment in H9c2 cells compared with those in the cells treated with DOX alone. These results were consistent with the aforementioned studies and further confirmed that the antioxidation of SS31 may serve crucial roles in the protection of DOX-induced $\mathrm{H} 9 \mathrm{c} 2$ cytotoxicity.

DOX has been reported to induce cardiomyocyte apoptosis by activating extrinsic and intrinsic apoptotic signaling, causing tissue injury $(39,40)$. Additionally, alleviating myocardial apoptosis potently suppresses the toxic effect of DOX on myocytes $(41,42)$. Consistent with previous studies, the results of the present study demonstrated that apoptosis-related proteins 
A

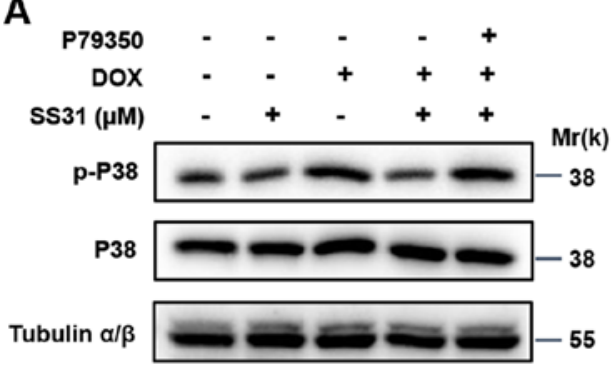

C

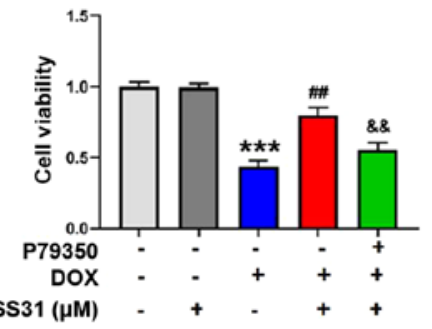

E

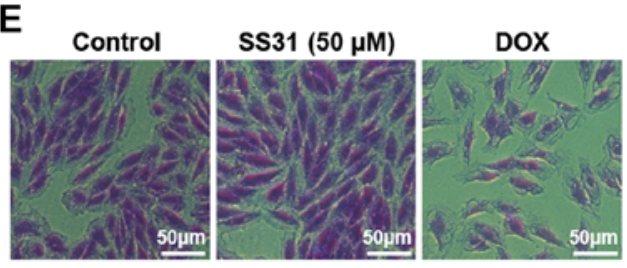

G Control

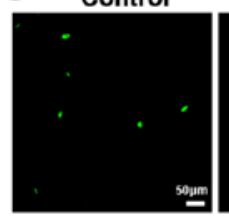

SS31 (50 $\mu \mathrm{M})$
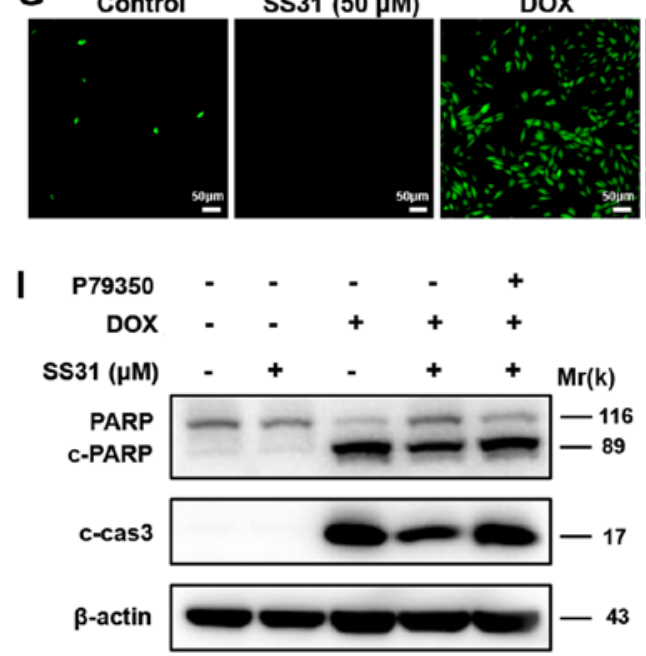

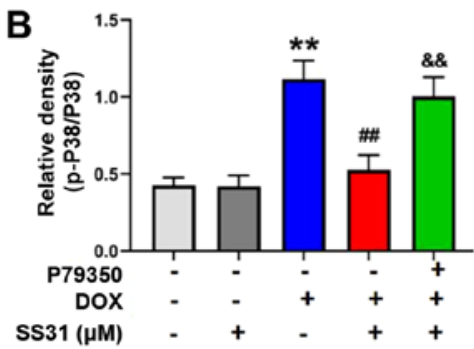

D

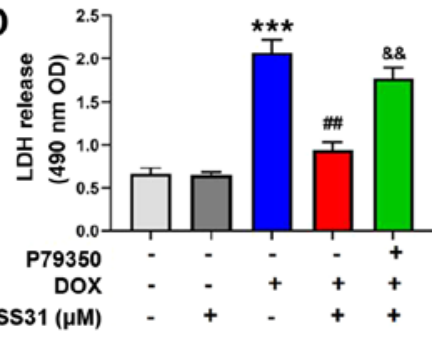

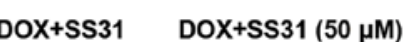
$(50 \mu \mathrm{M})$ +P79350

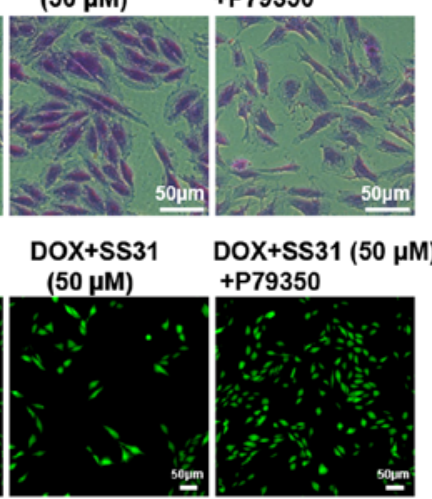

J

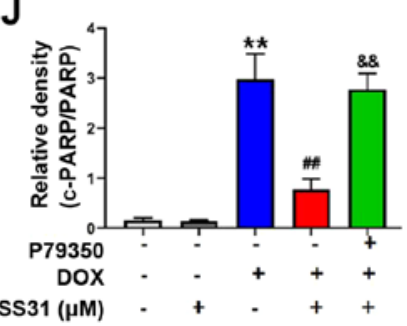

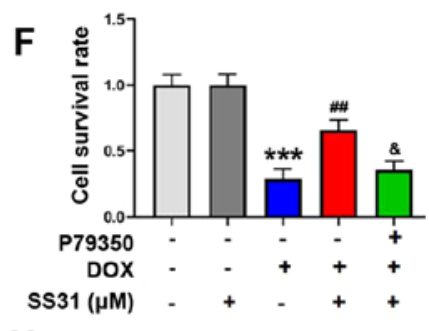

$\mathrm{H}$

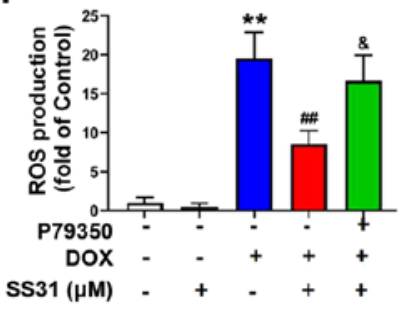

K

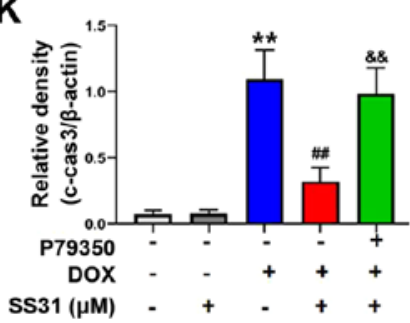

Figure 6. p38 agonist reverses the effects of SS31 in DOX-treated H9c2 cells. (A) H9c2 cells were treated with P79350 to activate the p38 signaling pathway. (B) Quantification of the western blot data. (C) H9c2 cell viability was detected following treatment with DOX, SS31 and P79350. (D) LDH release was detected in H9c2 cells. (E) Crystal violet staining of living cells. Magnification, x200. (F) Quantitative analysis of the crystal violet assay. (G) ROS contents were detected in H9c2 cells following treatment with DOX, SS31 and P79350. (H) Quantitative analysis of ROS contents. (I) The effects of P79350 on the levels of apoptosis-associated proteins in H9c2 cells treated with DOX and SS31. (J) Quantification of the PARP western blot bands. (K) Quantification of the c-cas3 western blot bands. ${ }^{* *} \mathrm{P}<0.01$ and ${ }^{* * *} \mathrm{P}<0.001$ vs. control; ${ }^{\# \#} \mathrm{P}<0.01$ vs. DOX; ${ }^{\&} \mathrm{P}<0.05$ and ${ }^{\& \&} \mathrm{P}<0.01$ vs. DOX $+\mathrm{SS} 31$. p-, phosphorylated; DOX, doxorubicin; SS31, Szeto-Schiller 31 peptide; LDH, lactate dehydrogenase; ROS, reactive oxygen species; PARP, poly(ADP-ribose) polymerase; OD, optical density; c-, cleaved; cas3, caspase-3.

were activated following DOX treatment, suggesting that DOX indeed induced apoptosis in H9c2 cells, whereas SS31 reduced the levels of proapoptotic proteins PARP, cleaved caspase- 3 and bax and enhanced the expression levels of the antiapoptotic protein bcl-2 compared with those in cells treated with DOX alone. Therefore, the antiapoptotic effect of SS31 may also play a key role in protecting DOX-induced cardiotoxicity.

The in vitro experiments in the present study demonstrated the antioxidant and antiapoptotic functions of SS31 in
DOX-treated H9c2 cells. The results of the in vivo experiments further confirmed the cardiotoxicity of DOX by histopathological analysis of mouse tissues, revealing notable fibrosis in the myocardial tissues of DOX-treated animals. Administration of SS31 partially reversed that detrimental changes, indicating that SS31 exerted a potential cardioprotective effect in vivo. Thus, the present study provided evidence that SS31 may protect the heart from DOX-induced toxicity in vitro and in vivo. 
The present study further assessed the underlying mechanism of the effects of SS31 in ameliorating DOX-induced toxicity in $\mathrm{H} 9 \mathrm{c} 2$ cells. As a member of the MAPK family, p38 MAPK is susceptible to various stimuli, such as oxidative stress, inflammatory cytokines, growth factors or high glucose levels. The canonical pathway of p38 MAPK activation is phosphorylation on a threonine and a tyrosine residue of the activation loop mediated by MAP2K (43). In cardiomyocytes, a previous study has demonstrated that the phosphorylation of p38 is triggered by hypoxia and exogenous $\mathrm{H}_{2} \mathrm{O}_{2}$ treatment (44). In the present study, DOX treatment significantly increased the phosphorylation of p38 MAPK compared with that in the control cells, whereas administration of SS31 inhibited this trend in $\mathrm{H} 9 \mathrm{c} 2$ cells. Notably, SS31 treatment exerted no significant effects on the expression and phosphorylation levels of ERK1/2 and JNK. A previous study has reported that SS31 peptide eliminates high glucose-induced mitochondrial oxidative stress by regulating the p38 MAPK signal pathway, which may be a novel therapeutic strategy to prevent hyperglycemia-induced neuronal perturbation (45). Another study has demonstrated that SS31 reduces the effects of sepsis-induced heart injury by inhibiting the p38 MAPK signaling pathway (12). The results of these studies were consistent with those of the present study. Therefore, SS31 may be a potent antioxidant peptide with a capacity for reducing ROS and inhibiting apoptosis, which may be associated with the inactivation of the p38 MAPK signaling pathway.

A previous study has mainly focused on the mechanism of DOX-induced cardiotoxicity and reported that high levels of mitochondrial ROS production are required for DOX-induced cardiac damage, and that the p38 MAPK signaling pathway is involved in DOX-induced cardiotoxicity (46). However, the aforementioned study has failed to provide the mechanism by which SS31 affects DOX-induced cardiomyocyte apoptosis. The present study demonstrated that SS31 may ameliorate DOX-induced ROS production and apoptosis in cardiomyocytes by inhibiting the activation of the p38 MAPK signaling pathway. To the best of the author's knowledge, this is the first report on the effects of SS31 on mitochondrial function describing the underlying mechanism to date.

Although the present study illustrated the anticardiotoxic effects of SS31 in DOX-induced injury models in vitro and in vivo, the study had certain limitations. It is necessary to evaluate the function of SS31 in other types of cells derived from heart, such as primary neonatal rat myocardial cells and AC16 cardiomyocytes. In addition, whether different types of modification affect the function of SS31 remains to be further verified. Therefore, our future studies will involve evaluating the effects of SS31 in multiple types of cardiomyocytes and performing modifications of SS31 to verify its cardioprotective function.

In summary, the results of the present study demonstrated that the mitochondria-targeted antioxidant peptide SS31 suppressed the generation of ROS and apoptosis by inhibiting the phosphorylation of p38 MAPK in DOX-treated H9c2 cells. In vivo, cotreatment with SS31 improved cardiac function and suppressed the occurrence of myocardial fibrosis induced by DOX compared with those in mice treated with DOX alone. Therefore, the results of the present study may provide a potential candidate molecule for the treatment of DOX-induced cardiotoxicity.

\section{Acknowledgements}

Not applicable.

\section{Funding}

This study was supported by grants from the National Natural Science Foundation of China (grant nos. 81570209 and 81873540) and The Key Clinical Frontier Technology Project of Department of Science and Technology of Jiangsu Provincial (grant no. BE2019752).

\section{Availability of data and materials}

The datasets used and/or analyzed during the current study are available from the corresponding author on reasonable request.

\section{Authors' contributions}

LZ and MF performed the experiments and wrote the manuscript. $\mathrm{XW}$ and $\mathrm{HZ}$ performed the cell experiments and participated in drafting the manuscript. JD performed a part of the in vivo study and participated in drafting the manuscript. LQ and ZC conceived, designed and supervised the project, and revised the manuscript. LZ and MF confirm the authenticity of all the raw data. All authors read and approved the final manuscript.

\section{Ethics approval and consent to participate}

All animal experiments were carried out in accordance with the Guide for the Care and Use of Laboratory and approved by the Institutional Animal Care and Use Committee of Nanjing Medical University (approval no. IACUC-1903030; Nanjing, China).

\section{Patient consent for publication}

Not applicable.

\section{Competing interests}

The authors declare that they have no competing interests.

\section{References}

1. Martins-Teixeira MB and Carvalho I: Antitumour anthracyclines: Progress and perspectives. ChemMedChem 15: 933-948, 2020.

2. Ji X, Ding W, Xu T, Zheng X, Zhang J, Liu M, Liu G and Wang J: MicroRNA-31-5p attenuates doxorubicin-induced cardiotoxicity via quaking and circular RNA Pan3. J Mol Cell Cardiol 140: 56-67, 2020.

3. Kalyanaraman B: Teaching the basics of the mechanism of doxorubicin-induced cardiotoxicity: Have we been barking up the wrong tree? Redox Biol 29: 101394, 2020.

4. Li DL and Hill JA: Cardiomyocyte autophagy and cancer chemotherapy. J Mol Cell Cardiol 71: 54-61, 2014.

5. Osataphan N, Phrommintikul A, Chattipakorn SC and Chattipakorn N: Effects of doxorubicin-induced cardiotoxicity on cardiac mitochondrial dynamics and mitochondrial function: Insights for future interventions. J Cell Mol Med 24: 6534-6557, 2020.

6. Octavia Y, Tocchetti CG, Gabrielson KL, Janssens S, Crijns HJ and Moens AL: Doxorubicin-induced cardiomyopathy: From molecular mechanisms to therapeutic strategies. J Mol Cell Cardiol 52: 1213-1225, 2012. 
7. Wang AJ, Zhang J, Xiao M, Wang S, Wang BJ, Guo Y, Tang Y and $\mathrm{Gu} \mathrm{J}$ : Molecular mechanisms of doxorubicin-induced cardiotoxicity: Novel roles of sirtuin 1-mediated signaling pathways. Cell Mol Life Sci: Jan 13, 2021 (Epub ahead of print). doi: 10.1007/ s00018-020-03729-y.

8. Tahover E, Segal A, Isacson R, Rosengarten O, Grenader T, Gips M, Cherny N, Heching NI, Mesika L, Catane R and Gabizon A Dexrazoxane added to doxorubicin-based adjuvant chemotherapy of breast cancer: A retrospective cohort study with a comparative analysis of toxicity and survival. Anticancer Drugs 28: 787-794, 2017

9. Ajith TA and Jayakumar TG: Mitochondria-targeted agents: Future perspectives of mitochondrial pharmaceutics in cardiovascular diseases. World J Cardiol 6: 1091-1099, 2014.

10. Lee FY, Shao PL, Wallace CG, Chua S, Sung PH, Ko SF, Chai HT, Chung SY, Chen KH, Lu HI, et al: Combined therapy with SS31 and mitochondria mitigates myocardial ischemia-reperfusion injury in rats. Int J Mol Sci 19: 2782, 2018.

11. Dai DF, Hsieh EJ, Chen T, Menendez LG, Basisty NB, Tsai L, Beyer RP, Crispin DA, Shulman NJ, Szeto HH, et al: Global proteomics and pathway analysis of pressure-overload-induced heart failure and its attenuation by mitochondrial-targeted peptides. Circ Heart Fail 6: 1067-1076, 2013.

12. Liu Y, Yang W, Sun X, Xie L, Yang Y, Sang M and Jiao R: SS31 ameliorates sepsis-induced heart injury by inhibiting oxidative stress and inflammation. Inflammation 42: 2170-2180, 2019.

13. Dai DF, Chen T, Szeto H, Nieves-Cintrón M, Kutyavin V, Santana LF and Rabinovitch PS: Mitochondrial targeted antioxidant Peptide ameliorates hypertensive cardiomyopathy. J Am Coll Cardiol 58: 73-82, 2011.

14. Yue J and Lopez JM: Understanding MAPK signaling pathways in apoptosis. Int J Mol Sci 21: 2346, 2020.

15. Yue TL, Wang C, Gu JL, Ma XL, Kumar S, Lee JC, Feuerstein GZ, Thomas H, Maleeff B and Ohlstein EH: Inhibition of extracellular signal-regulated kinase enhances ischemia/reoxygenation-induced apoptosis in cultured cardiac myocytes and exaggerates reperfusion injury in isolated perfused heart. Circ Res 86: 692-699, 2000.

16. Wang N, Guan P, Zhang JP, Li YQ, Chang YZ, Shi ZH, Wang FY and Chu L: Fasudil hydrochloride hydrate, a Rho-kinase inhibitor, suppresses isoproterenol-induced heart failure in rats via JNK and ERK1/2 pathways. J Cell Biochem 112: 1920-1929, 2011.

17. Liu D, Ma Z, Di S, Yang Y, Yang J, Xu L, Reiter RJ, Qiao S and Yuan J: AMPK/PGC1 $\alpha$ activation by melatonin attenuates acute doxorubicin cardiotoxicity via alleviating mitochondrial oxidative damage and apoptosis. Free Radic Biol Med 129: 59-72, 2018

18. Krais JJ and Johnson N: Ectopic RNF168 expression promotes break-induced replication-like DNA synthesis at stalled replication forks. Nucleic Acids Res 48: 4298-4308, 2020.

19. Fan J, Shen W, Lee SR, Mathai AE, Zhang R, Xu G and Gillies MC: Targeting the Notch and TGF- $\beta$ signaling pathways to prevent retinal fibrosis in vitro and in vivo. Theranostics 10 : 7956-7973, 2020.

20. National Research Council (US): Committee for the Update of the Guide for the Care and Use of Laboratory Animals: Guide for the Care and Use of Laboratory Animals. 8th edition. National Academies Press, Washington, DC, 2011.

21. Oh J, Lee BS, Lim G, Lim H, Lee CJ, Park S, Lee SH, Chung JH and Kang SM: Atorvastatin protects cardiomyocyte from doxorubicin toxicity by modulating survivin expression through FOXO1 inhibition. J Mol Cell Cardiol 138: 244-255, 2020.

22. Zhang L, Wang X, Feng M, Zhang H, Xu J, Ding J, Cheng Z and Qian L: Peptidomics analysis reveals peptide PDCryab1 inhibits doxorubicin-induced cardiotoxicity. Oxid Med Cell Longev 2020: 7182428, 2020.

23. Liang L, Tu Y, Lu J, Wang P, Guo Z, Wang Q, Guo K, Lan R, Li H and Liu P: Dkk1 exacerbates doxorubicin-induced cardiotoxicity by inhibiting the Wnt/ $\beta$-catenin signaling pathway. J Cell Sci 132: cs228478, 2019.

24. Rochette L, Guenancia C, Gudjoncik A, Hachet O, Zeller M, Cottin Y and Vergely C: Anthracyclines/trastuzumab: New aspects of cardiotoxicity and molecular mechanisms. Trends Pharmacol Sci 36: 326-348, 2015.

25. Hantson P: Mechanisms of toxic cardiomyopathy. Clin Toxicol (Phila) 57: 1-9, 2019.

26. Zhao L, Qi Y, Xu L, Tao X, Han X, Yin L and Peng J: MicroRNA-140-5p aggravates doxorubicin-induced cardiotoxicity by promoting myocardial oxidative stress via targeting Nrf2 and Sirt2. Redox Biol 15: 284-296, 2018.

27. Zhang P, Chen Z, Lu D, Wu Y, Fan M, Qian J and Ge J: Overexpression of COX5A protects $\mathrm{H} 9 \mathrm{c} 2$ cells against doxorubicin-induced cardiotoxicity. Biochem Biophys Res Commun 524: 43-49, 2020.
28. Kim HS, Lee YS and Kim DK: Doxorubicin exerts cytotoxic effects through cell cycle arrest and Fas-mediated cell death. Pharmacology 84: 300-309, 2009.

29. Yu J, Gao H, Wu C, Xu QM, Lu JJ and Chen X: Diethyl blechnic, a novel natural product isolated from salvia miltiorrhiza bunge, inhibits doxorubicin-induced apoptosis by inhibiting ROS and activating JNK1/2. Int J Mol Sci 19: 1809, 2018.

30. Varela-Lopez A, Battino M, Navarro-Hortal MD, Giampieri F, Forbes-Hernández TY, Romero-Márquez JM, Collado R and Quiles JL: An update on the mechanisms related to cell death and toxicity of doxorubicin and the protective role of nutrients. Food Chem Toxicol 134: 110834, 2019.

31. Gorini S, De Angelis A, Berrino L, Malara N, Rosano G and Ferraro E: Chemotherapeutic drugs and mitochondrial dysfunction: Focus on doxorubicin, trastuzumab, and sunitinib. Oxid Med Cell Longev 2018: 7582730, 2018.

32. Orrenius S, Gogvadze V and Zhivotovsky B: Mitochondrial oxidative stress: Implications for cell death. Annu Rev Pharmacol Toxicol 47: 143-183, 2007.

33. Wallace KB, Sardao VA and Oliveira PJ: Mitochondrial determinants of doxorubicin-induced cardiomyopathy. Circ Res 126: 926-941, 2020.

34. Mitchell W, Ng EA, Tamucci JD, Boyd KJ, Sathappa M, Coscia A, Pan M, Han X, Eddy NA, May ER, et al: The mitochondria-targeted peptide SS-31 binds lipid bilayers and modulates surface electrostatics as a key component of its mechanism of action. J Biol Chem 295: 7452-7469, 2020.

35. Hou Y, Shi Y, Han B, Liu X, Qiao X, Qi Y and Wang L: The antioxidant peptide SS31 prevents oxidative stress, downregulates CD36 and improves renal function in diabetic nephropathy. Nephrol Dial Transplant 33: 1908-1918, 2018.

36. Reddy PH, Manczak M, Yin X and Reddy AP: Synergistic protective effects of mitochondrial division inhibitor 1 and mitochondria-targeted small peptide SS31 in Alzheimer's disease. J Alzheimers Dis 62: 1549-1565, 2018.

37. Zhou J, Li Z, Chen Z and Yang K: Protective effect of mitochondria-targeted antioxidant SS31 on early brain injury following subarachnoid hemorrhage in rats. Zhong Nan Da Xue Xue Bao Yi Xue Ban 42: 1003-1009, 2017 (In Chinese).

38. Yin X, Manczak M and Reddy PH: Mitochondria-targeted molecules MitoQ and SS31 reduce mutant huntingtin-induced mitochondrial toxicity and synaptic damage in Huntington's disease. Hum Mol Genet 25: 1739-1753, 2016

39. Ma H, Chen S, Xiong H, Wang M, Hang W, Zhu X, Zheng Y, $\mathrm{Ge}$ B, Li R and Cui H: Astaxanthin from Haematococcus pluvialis ameliorates the chemotherapeutic drug (doxorubicin) induced liver injury through the Keap1/Nrf2/HO-1 pathway in mice. Food Funct 11: 4659-4671, 2020.

40. Vu M, Kassouf N, Ofili R, Lund T, Bell C and Appiah S: Doxorubicin selectively induces apoptosis through the inhibition of a novel isoform of $\mathrm{Bcl} 2$ in acute myeloid leukaemia MOLM13 cells with reduced Beclin 1 expression. Int J Oncol 57: 113-121, 2020.

41. Faridvand Y, Haddadi P, Vahedian V, Nozari S, Nejabati HR, Pezeshkian M, Afrasiabi A, Safaie N, Jodati A and Nouri M: Human amnion membrane proteins prevent doxorubicin- induced oxidative stress injury and apoptosis in rat $\mathrm{H} 9 \mathrm{c} 2$ cardiomyocytes. Cardiovasc Toxicol 20: 370-379, 2020.

42. Wu YZ, Zhang L, Wu ZX, Shan TT and Xiong C: Berberine ameliorates doxorubicin-induced cardiotoxicity via a SIRT1/p66Shc-mediated pathway. Oxid Med Cell Longev 2019: $2150394,2019$.

43. Cuadrado A and Nebreda AR: Mechanisms and functions of p38 MAPK signalling. Biochem J 429: 403-417, 2010.

44. Kulisz A, Chen N, Chandel NS, Shao Z and Schumacker PT: Mitochondrial ROS initiate phosphorylation of p38 MAP kinase during hypoxia in cardiomyocytes. Am J Physiol Lung Cell Mol Physiol 282: L1324-L1329, 2002.

45. Cao M, Jiang J, Du Y and Yan P: Mitochondria-targeted antioxidant attenuates high glucose-induced P38 MAPK pathway activation in human neuroblastoma cells. Mol Med Rep 5: 929-934, 2012

46. Guo Z, Tang N, Liu FY, Yang Z, Ma SQ, An P, Wu HM, Fan D and Tang QZ: TLR9 deficiency alleviates doxorubicin- induced cardiotoxicity via the regulation of autophagy. J Cell Mol Med 24: 10913-10923, 2020.

This work is licensed under a Creative Commons Attribution-NonCommercial-NoDerivatives 4.0 International (CC BY-NC-ND 4.0) License. 